\title{
MAGIC observations of the nearby short GRB 160821B: implications for afterglow physics and gravitational wave follow-up
}

\author{
Susumu Inoue ${ }^{* 1}$, Lara Nava ${ }^{2,3}, \mathrm{Koji} \mathrm{Noda}^{4}$, Pierre Colin $^{5}$, Satoshi Fukami ${ }^{4}$, \\ Alessandro Carosi $^{3,6}$, Alessio Berti ${ }^{7}$, Michele Palatiello ${ }^{2}$, Francesco Longo ${ }^{2,8}$, for \\ the MAGIC Collaboration ${ }^{\dagger}$
}

E-mail: susumu.inouedriken.jp

${ }^{1}$ iTHEMS, RIKEN, Wako, Japan; ${ }^{2}$ U. Udine and INFN Trieste, Udine, Italy; ${ }^{3}$ INAF, Rome, Italy;

${ }^{4}$ ICRR, U. Tokyo, Kashiwa, Japan; ${ }^{5}$ MPP, Munich, Germany; ${ }^{6}$ LAPP, Annecy, France $;{ }^{7}$ INFN,

Rome, Italy; ${ }^{8} \mathrm{U}$. Trieste, Trieste, Italy

\begin{abstract}
Gamma-ray bursts (GRBs) are the most luminous sources of electromagnetic radiation in the Universe, many of whose fundamental aspects are still mysterious. Aiming to clarify their physical origin by detecting them in very-high-energy (VHE; $>0.1 \mathrm{TeV}$ ) gamma rays, the MAGIC telescopes have been conducting fast, automatic follow-up of GRBs as a key observation program. GRB 160821B, one of the nearest short GRBs known at $z \sim 0.16$ with evidence of a coincident kilonova, was followed up, starting from $24 \mathrm{sec}$ after the burst trigger. Despite non-optimal observing conditions, hints of a gamma-ray signal are found above $\sim 500 \mathrm{GeV}$ a few hours after the burst. Assuming that the signal was real, we interpret the multi-wavelength observations of this GRB in terms of standard afterglow models. The potential VHE signal can be consistently explained as synchrotron-self-Compton emission from electrons accelerated in the external forward shock with a plausible set of parameters. We also discuss the expectations when such afterglow emission is observed at large angles from the GRB jet axis, corresponding to VHE follow-up of gravitational wave alerts for neutron star (NS) mergers. Interesting prospects are found for sufficiently nearby events with current imaging atmospheric Cherenkov telescopes. Future VHE observations of short GRBs as well as GW sources may provide important new insight into the physics of NS mergers and related phenomena.
\end{abstract}

36th International Cosmic Ray Conference -ICRC2019-

July 24th - August 1st, 2019

Madison, WI, U.S.A.

\footnotetext{
* Speaker.

† https://magic.mpp.mpg. de/. For collaboration list see PoS(ICRC2019)1177.
} 
Gamma-ray bursts (GRBs) are the most luminous sources of electromagnetic radiation known in the Universe. However, many fundamental aspects of their physical origin remain poorly understood. Aiming to clarify the underlying physical mechanisms through their detection in gamma rays at very-high-energy (VHE; $>0.1 \mathrm{TeV}$ ), the MAGIC telescopes have been regularly conducting fast, automatic follow-up observations of GRBs. As part of this key program, GRB 160821B [四] was followed up, starting from $24 \mathrm{sec}$ after the burst trigger. GRB 160821B is particularly noteworthy in being one of the nearest short-duration GRBs known at $z \sim 0.16$, with evidence of

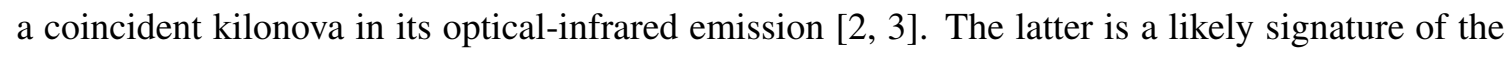
synthesis and ejection of heavy (r-process) elements during the merging of binary neutron stars (NSs). Despite challenging observing conditions with bright Moon and non-optimal weather, hints of a gamma-ray signal are found above $\sim 500 \mathrm{GeV}$ a few hours after the burst.

Assuming that the signal was real, we interpret these observations together with those at other wavelengths in terms of standard GRB afterglow models. Utilizing a numerical code that allows a comprehensive parameter search, the potential VHE signal can be consistently explained as synchrotron-self-Compton (SSC) emission from electrons accelerated in the external forward shock with a plausible set of parameters. Interesting inferences can be deduced for some quantities that had been poorly constrained, such as the number fraction of electrons channeled into a non-thermal population.

The afterglows of short GRBs below the X-ray band can be complicated by various emission components superposed on the synchrotron emission from the forward shock. Besides kilonovae at optical-infrared wavelengths, these include "extended" emission in X-rays (most likely related to long-lasting activity of the central engine), and reverse shock emission in the radio to optical bands. For GRB 160821B, all of these components appear to be present [ [, B] ], making difficult a robust interpretation of the forward shock afterglow emission. This in turn can imply significant uncertainties in the characterization of the kilonova component and associated quantities such as the mass, velocity and elemental composition of the merger ejecta. Future VHE observations of short GRBs can play an important role in improving such measurements, through detections of the SSC component that would allow more robust determinations of the forward shock emission.

Furthermore, we discuss the expectations when such afterglow emission is observed at large angles from the GRB jet axis, corresponding to VHE follow-up of gravitational wave (GW) alerts for NS mergers. We find that events with distances and viewing angles similar to GW170817 may be detectable with current imaging atmospheric Cherenkov telescopes. Such observations may reveal important new insight into the off-axis structure of jets produced by NS mergers.

\section{References}

[1] D. M. Palmer et al., GRB Coordinates Network, Circular 19844 (2016).

[2] E. Troja et al., MNRAS, submitted, arXiv:1905.01290.

[3] G. P. Lamb et al., ApJ, submitted, arXiv:1905.02159. 